\title{
PAPULONODULAR MUCINOSIS IN A PATIENT WITH SJOGREN SYNDROME
} Camila da Silva Cendon Durann,, ${ }^{1, *}$ Lucas Brandão Araujo da Silva ${ }^{1}$, Osvaldo Luiz Camata Junior ${ }^{1}$, Sandra Gofinet Pasoto $^{1}$,
Eduardo Ferreira Borba Neto ${ }^{1}$

1. Universidade de São Paulo, São Paulo (SP), Brazil.

*Corresponding author: milla_duran@hotmail.com

\section{BACKGROUND}

Cutaneous mucinosis is a group of disorders involving mucin deposition in the skin and its adnexa. Mucin is a jelly-like substance composed of acid glycosaminoglycans (GAGs) produced by skin fibroblasts. It is suggested that circulating autoantibodies and increased cytokines are capable of stimulating skin fibroblasts in connective tissue diseases (CTDs), leading to excess GAGs synthesis. We will describe an atypical case of papulonodular cutaneous mucinosis associated with Sjogren's Syndrome.

\section{CASE REPORT}

A 52-year-old woman with a 1-year history of skin-colored papules and nodules, measuring about 2-5 mm in the trunk, abdomen, upper and lower limbs (Fig. 1), associated with erythematoviolaceous lesions on her back and lower limbs. She also referred arthralgia in wrists and MCFs, xerostomia and episodes of parotitis. Physical examination was normal, except for the skin lesions mentioned above. Diagnostic work-up revealed a normal blood count, CRP, ESR, renal and thyroid function. Protein electrophoresis showed polyclonal hypergammaglobulinemia (gamma $1.9 \mathrm{~g} / \mathrm{dL}$ ). Serologies were negative. Immunologic studies revealed ANA 1:320 with a speckled pattern, Anti-Ro > $200 \mathrm{U} / \mathrm{mL}$, anti-La $19.7 \mathrm{U} / \mathrm{mL}$ (inconclusive), RF: $39.6 \mathrm{UI} / \mathrm{mL}(\mathrm{NR}<14$ ). Anti-DNA, anti-Sm, ANCA, and lupus anticoagulant were negative. Complement levels, urianalysis and proteinuria were normal. Scintigraphy of salivary glands showed a deficit in salivary function. Histopathological analysis of papulonodular lesions showed abundant deposition of mucin in the dermis in addition to a discrete and perivascular lymphohistiocytic infiltrate. Erythematoviolaceous lesions on her back showed leukocytoclastic vasculitis. Based on clinical and laboratory data, a diagnosis of Sjogren's syndrome was made. She had been using hydroxychloroquine (400 mg/day) with no improvement, referring accelerated appearance of lesions. Azathioprine $100 \mathrm{mg} / \mathrm{day}$ was prescribed, with improvement in arthralgia and stabilization of skin lesions.

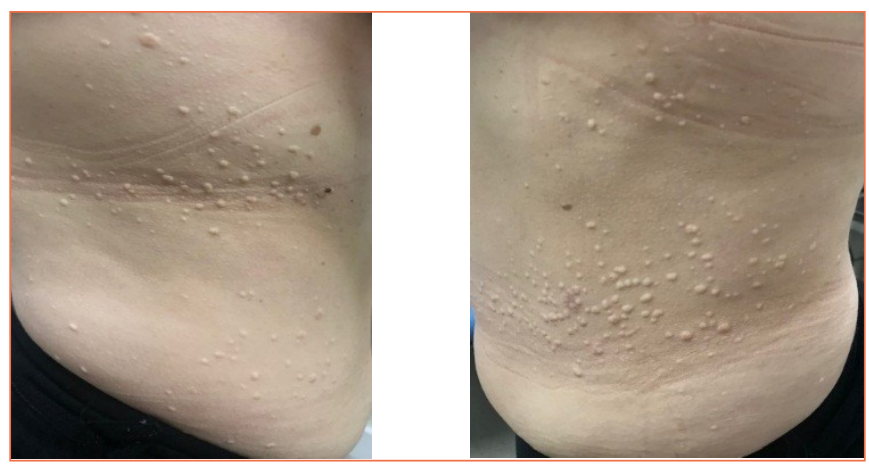

Figure 1. Skin-colored papulonodular lesions on trunk and abdomen.

\section{CONCLUSION}

Cutaneous mucinosis associated with CTDs can be primary or secondary. When secondary, mucin deposition is usually subclinical and microscopic and is accompanied by histologic features of the underlying disease. In the histology of primary mucinosis, mucin deposition predominates, leading to clinically evident skin lesion. 
Papulonodular mucinosis is well described in SLE, with an estimated prevalence of $1.5 \%$. There are also reports of cutaneous mucinosis in rheumatoid arthritis, MCTD, systemic sclerosis and dermatomyositis. Sjogren's syndrome has already been reported in association with ichthyosiform mucinosis simulating pretibial myxedema, but as far as we know it has never been reported in association with papulonodular mucinosis. There is no consensus about the treatment. Topical corticosteroids, hydroxychloroquine, methotrexate and azathioprine has been used with variable responses. 